\title{
Review Article \\ Worldwide Dissemination of the NDM-Type Carbapenemases in Gram-Negative Bacteria
}

\author{
Laurent Dortet, ${ }^{1}$ Laurent Poirel, ${ }^{1,2}$ and Patrice Nordmann ${ }^{1,2}$ \\ ${ }^{1}$ INSERM U914 "Emerging Resistance to Antibiotics", 78 Avenue du Général Leclerc, 94270 Le Kremlin-Bicêtre, France \\ ${ }^{2}$ Medical and Molecular Microbiology Unit, Department of Medicine, Faculty of Science, University of Fribourg, \\ 3 Rue Albert Gockel, 1700 Fribourg, Switzerland
}

Correspondence should be addressed to Patrice Nordmann; patrice.nordmann@unifr.ch

Received 7 December 2013; Accepted 15 February 2014; Published 26 March 2014

Academic Editor: Karmen Torkar

Copyright (C) 2014 Laurent Dortet et al. This is an open access article distributed under the Creative Commons Attribution License, which permits unrestricted use, distribution, and reproduction in any medium, provided the original work is properly cited.

\begin{abstract}
The emergence of one of the most recently described carbapenemases, namely, the New Delhi metallo-lactamase (NDM-1), constitutes a critical and growingly important medical issue. This resistance trait compromises the efficacy of almost all lactams (except aztreonam), including the last resort carbapenems. Therapeutical options may remain limited mostly to colistin, tigecycline, and fosfomycin. The main known reservoir of NDM producers is the Indian subcontinent whereas a secondary reservoir seems to have established the Balkans regions and the Middle East. Although the spread of bla $a_{\mathrm{NDM}}$-like genes (several variants) is derived mostly by conjugative plasmids in Enterobacteriaceae, this carbapenemase has also been identified in P. aeruginosa and Acinetobacter spp. Acinetobacter sp. may play a pivotal role for spreading $b l a_{\mathrm{NDM}}$ genes for its natural reservoir to Enterobacteriaceae. Rapid diagnostic techniques (Carba NP test) and screening of carriers are the cornerstone to try to contain this outbreak which threatens the efficacy of the modern medicine.
\end{abstract}

\section{Introduction}

During the last decade the emergence of carbapenemaseproducing strains among Enterobacteriaceae, Pseudomonas spp., and Acinetobacter baumannii is remarkable. A variety of carbapenemases have been reported such as the Ambler class A KPC-type (mostly identified in Enterobacteriaceae and Pseudomonas aeruginosa) and GES-type (mostly in A. baumannii), the Ambler class B metallo- $\beta$-lactamases (MBL) of VIM-, IMP-, GIM-, and NDM-types, and the Ambler class D carbapenemases of the OXA-48 type in Enterobacteriaceae and of OXA-23, OXA-24/-40, OXA-58, and OXA-143 types in Acinetobacter spp. The emergence of the most recently described carbapenemase, namely, the New Delhi metallo$\beta$-lactamase (NDM-1), constitutes a critical medical issue. Indeed, this enzyme compromises the efficacy of almost all $\beta$-lactams (except aztreonam), including the last resort carbapenems. Although most of the NDM-producing strains identified are Enterobacteriaceae, this carbapenemase has also been reported from Acinetobacter spp. and more rarely from $P$. aeruginosa, both species causing severe nosocomial infections, including urinary tract infections, peritonitis, septicemia, and pulmonary infections. The Indian subcontinent, the Balkans regions, and the Middle East are considered to be the main reservoirs of NDM producers. Since therapeutical options are limited to very few antibiotics such as colistin, tigecycline, and fosfomycin, hospital- and communityacquired infections caused by NDM-1 producers are difficult to eradicate. Isolation of infected patients and carriers and rapid diagnostic techniques are the key factors that contribute to contain this outbreak that threatens the efficacy of the modern medicine.

\section{Clinical Impact of the Antibiotic Resistance Patterns of NDM Producers for the Treatment}

Currently, one of the most clinically significant carbapenemase is the recently described NDM-1 (New Delhi metallo$\beta$-lactamase). This carbapenemase belongs to the class B of Ambler $\beta$-lactamases classification that includes the metallo$\beta$-lactamases (MBLs). NDM-1 shares very little identity with 

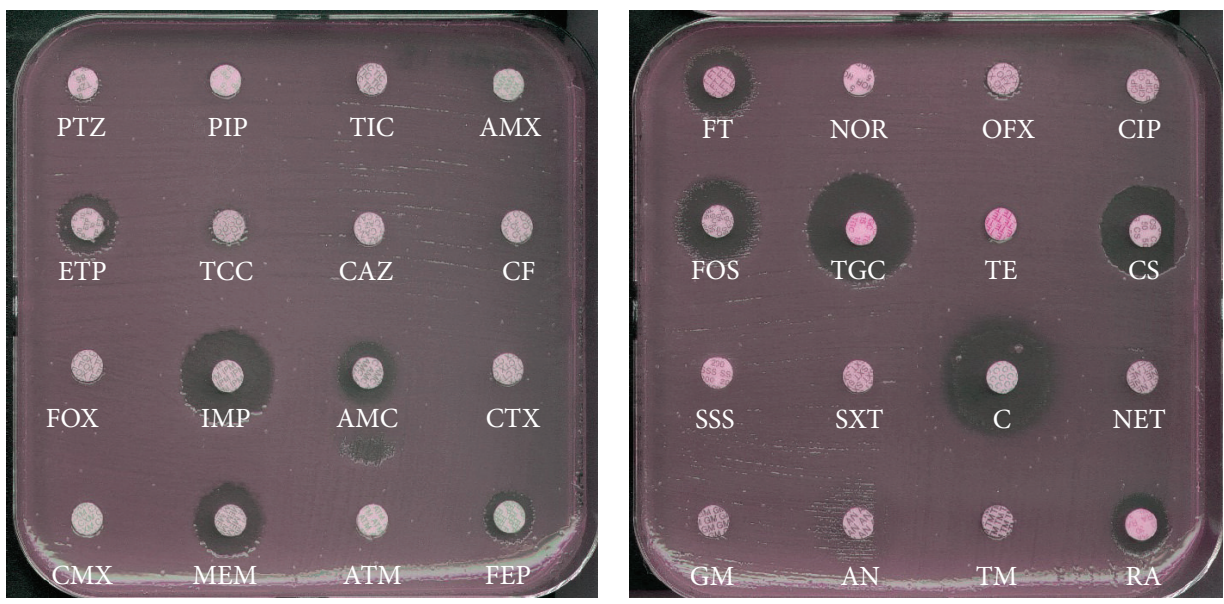

FIGURE 1: Antibiogram of a NDM-1-producing K. pneumoniae isolate. The $b l a_{\mathrm{NDM}-1}$ gene was located onto a IncHIIB plasmid of ca. $\sim 200 \mathrm{~kb}$ in that strain that also harbored two additional $\beta$-lactamase genes $\left(b l a_{\mathrm{CTX}-\mathrm{M}-15}, b l a_{\mathrm{SHV}-12}, b l a_{\mathrm{OXA}-1}\right)$ and an aminoglycoside methylase $(\operatorname{armA})$ responsible for high-level resistance to all aminoglycosides. PTZ, piperacillin + tazobactam; PIP, piperacillin; TIC, ticarcillin; AMX, amoxicillin; ETP, ertapenem; TCC, ticarcillin + clavulanic acid; CAZ, ceftazidime; CF, cefalotin; FOX, cefoxitin; IMP, imipenem; AMC, amoxicillin + clavulanic acid; CTX, cefotaxime; CMX, cefuroxime; MEM, meropenem; ATM, aztreonam; FEP, cefepime; FT, nitrofurantoin; NOR, norfloxacin; OFX, ofloxacin; CIP, ciprofloxacin; FOS, fosfomycin; TGC, tigecycline; TE, tetracycline; CS, colistin; SSS, sulfonamide; SXT, sulfamethoxazole + trimethoprim; C, chloramphenicol; NET, netilmicin; GM, gentamicin; AN, amikacin; TM, tobramycin; RA, rifampicin.

other MBLs, the most similar being VIM-1/VIM-2 with only $32.4 \%$ amino acid identity. Compared to VIM-2, NDM-1 displays tighter binding to most cephalosporins, in particular to cefuroxime $\left(K_{\mathrm{mNDM}-1}=8 \mu \mathrm{M}, K_{\mathrm{mVIM}-2}=22 \mu \mathrm{M}\right)$, cefotaxime $\left(K_{\mathrm{mNDM}-1}=10 \mu \mathrm{M}, K_{\mathrm{mVIM}-2}=32 \mu \mathrm{M}\right)$, cephalothin $\left(K_{\mathrm{mNDM}-1}=10 \mu \mathrm{M}, K_{\mathrm{mVIM}-2}=44 \mu \mathrm{M}\right)$, and penicillins $\left(K_{\mathrm{mNDM}-1}=16 \mu \mathrm{M}, K_{\mathrm{mVIM}-2}=49 \mu \mathrm{M}\right)$. Like all other MBLs, NDM-1 efficiently hydrolyses a broad range of $\beta$-lactams including penicillins, cephalosporins, and carbapenems, just sparing monobactams such as aztreonam. NDM-1 does not bind to carbapenems as tightly as IMP-1 or VIM-2 does, and the turnover rate of carbapenem hydrolysis is similar to that of VIM-2 $\left(k_{\text {cat }} / K_{\mathrm{m}}\right.$ are $0.21,1.2$, and $0.99 \mathrm{~s}^{-1} \cdot \mu \mathrm{M}^{-1}$ for NDM-1, IMP-1, and VIM-2, resp.). Similar to the other MBLs, the active site of NDM-1 contains two metal ion binding sites: the His and Cys sites. Accordingly, a 3D-structure modelling of the NDM-1 enzyme showed that two zinc ions were present at both the His and Cys sites with a distance of $4.20 \AA$ [1]. Indeed, the hydrolysis activity of MBLs depends on the interaction of the $\beta$-lactam molecule with $\mathrm{Zn}^{2+}$ ion(s) in their active site. Consequently, their activity is inhibited by chelators of divalent cations, such as EDTA. Accordingly, the efficacy of EDTA (Ca-EDTA) has been evaluated in a mouse model of sepsis caused by an NDM-1-producing Escherichia coli. It has been shown that a combination therapy using imipenem/cilastatin sodium (IPM/CS) and Ca-EDTA reduced the bacterial inoculum, as compared to IPM/CS alone suggesting the possibility to use Ca-EDTA in clinical therapeutics [2]. Comparison of IMP-1, VIM-2, and NDM-1 by an in silico approach revealed that NDM-1 might have greater drug profile and catalytic efficiency than IMP-1 and VIM-2 due to a larger pocket opening and a lower distance between the $\mathrm{Zn}-\mathrm{I}$ ion and $\beta$-lactam oxygen of the carbapenem [3].
It is noteworthy that a quite systematic association with other antibiotic resistance determinants is observed in almost all NDM producers (Enterobacteriaceae, Acinetobacter, and Pseudomonas). Those associated resistance determinants are AmpC cephalosporinases, clavulanic acid inhibited expanded-spectrum $\beta$-lactamases (ESBLs), other types of carbapenemases (OXA-48-, VIM-, and KPC-types), and resistance to aminoglycosides (16S RNA methylases), to quinolones (Qnr), to macrolides (esterases), to rifampicin (rifampicin-modifying enzymes), to chloramphenicol, and to sulfamethoxazole [4-9]. Consequently, most of the NDM-1 producers remain susceptible only to two bactericidal antibiotics (colistin and fosfomycin) and a single bacteriostatic antibiotic (tigecycline) [10, 11] (Figure 1). In vitro synergy combination assays performed with NDM-1 producers with those three antibiotic molecules showed a synergistic activity of colistin and fosfomycin, of colistin and tigecycline in rare cases, whereas most of the antibiotic associations remain neutral for most of the tested isolates [12]. Since NDM-1 does not hydrolyze aztreonam, a combination therapy including aztreonam and avibactam (also named NXL-104), a novel serine $\beta$-lactamase inhibitor inhibiting the most frequent broadspectrum hydrolyzing- $\beta$-lactamases hydrolyzing aztreonam has been suggested as a possible strategy against NDM-1producing Enterobacteriaceae. This therapeutic option seems to be a very efficient combination therapy in vitro $[13,14]$.

\section{Infections Caused by NDM Producers}

Since NDM producers were mainly described in Enterobacteriaceae, infections caused by NDM producers include urinary tract infections, peritonitis, septicemia, pulmonary infections, soft tissue infections, and device-associated infections. As observed for other multidrug-resistant bacteria, 
it is highly probable that colonization of the gut flora might precede the infection by NDM producers and orofecal transmission in the community might occur mostly through hand contamination, food, and water. Among the NDM1-producing Enterobacteriaceae, Klebsiella pneumoniae and E. coli are the most often described species. Both hospitaland community-acquired infections have been reported. However, this carbapenemase is also frequently described in other enterobacterial species including Klebsiella oxytoca, Enterobacter cloacae, Citrobacter freundii, Proteus mirabilis, Salmonella spp., and Providencia spp. Although most of NDM-producing bacteria are Enterobacteriaceae, this carbapenemase was also reported from Acinetobacter spp. [15$32]$ and in rare cases Pseudomonas aeruginosa [33, 34].

Since, no specific virulence factor is known to be associated with $b l a_{\mathrm{NDM}-1}$-carrying plasmids $[6,35-39]$, there is no evidence that NDM-producing bacteria are more virulent than other strains [40-42]. However, some rare isolates of NDM-1-producing virulent enteric bacteria such as Salmonella [43-45] and Vibrio cholerae [46, 47] have been described.

\section{Epidemiology of NDM-Producing Bacteria}

NDM-1 was first identified in 2008 in a K. pneumoniae isolate recovered from a Swedish patient who has been previously hospitalized in New Delhi, India [48]. Since then, NDM carbapenemases are the focus of worldwide attention due to the rapid dissemination of the corresponding gene among Enterobacteriaceae and Acinetobacter spp. mainly (Figure 2). Rapidly, a link between NDM-producing Enterobacteriaceae and the Indian subcontinent has been pointed out [49-51], and prevalence rates of NDM-producing Enterobacteriaceae were found to range from 5 to $18.5 \%$ in Indian and Pakistan hospitals [52-55]. In addition, the $b l a_{\mathrm{NDM}-1}$ gene was detected not only in patient samples, but also in drinking water and seepage samples in New Delhi [47]. The occurrence of NDM1-producing bacteria in environmental samples in New Delhi is significant for people living in the city who often rely onto public water and poor sanitation facilities. A secondary reservoir of NDM-1 producers was then highlighted through several studies reporting patients colonized or infected with NDM-1 producers originating from the Balkan states [50, 5661]. Recent reports also suggested that the Middle East might be an additional reservoir of NDM producers [62-67]. This dissemination of NDM producers in the Middle East could mostly be linked to the population exchange between the Middle East and the Indian subcontinent. However, NDM-1 producing bacteria have now been reported worldwide with a rapid dissemination from the two previously described reservoirs, namely, the Indian subcontinent and the Balkan countries.

As observed with the dissemination of NDM-1-producing Enterobacteriaceae, NDM-producing Acinetobacter has also been recovered from environmental samples in China [31]. Currently, the majority of NDM-producing Acinetobacter spp. are reported from China $[18,19,25,29-32]$ and Middle East $[17,20-24,26]$.

\section{Genetic Features of the $b l a_{\text {NDM }}$ Genes}

In Enterobacteriaceae, the $b l a_{\mathrm{NDM}-1}$ gene is located mostly onto conjugative plasmids belonging to several incompatibility groups $[6,7,25,35,37,38,63,68]$. However, investigation of a worldwide collection of NDM-1-producing enterobacterial isolates showed that the current spread of the $b l a_{\mathrm{NDM}-1}$ gene is not related to the spread of specific clones, specific plasmids, or single genetic structure [7]. In Acinetobacter spp. the bla $a_{\mathrm{NDM}^{-}}$-type genes are found to be either plasmid- or chromosome-located, and in the rare NDM-1-producing $P$. aeruginosa, the $b l a_{\mathrm{NDM}-1}$ gene was found to be chromosomally located [33,34]. Investigations on the immediate genetic environment of $b{ }_{\mathrm{NDM}}$ genes revealed the presence of a conserved structure that always associated the complete or truncated insertion sequence ISAba125 at the $5^{\prime}$-end and the $b l e_{\mathrm{MBL}}$ gene (encoding resistance to the anticancer drug bleomycin) at the $3^{\prime}$-end of the $b l a_{\mathrm{NDM}}$ genes [69] (Figure 3). In addition, in several studies focusing on NDM-producing $A$. baumannii, the $b l a_{\mathrm{NDM}}$ gene was located between two copies of the ISAba125 element, forming a composite transposon named Tn125 [15-17, 20, 24, 26, 70, 71] (Figure 3). Systematic identification of a truncated form of this composite transposon in Enterobacteriaceae, while it was described in its entire form in A. baumannii, strongly suggesting that Acinetobacter spp. has been a reservoir of those $b l a_{\mathrm{NDM}}$ genes before targeting enterobacterial species. Those findings highlight that even though A. baumannii is usually recognized as a final acceptor for resistance genes, it may acquire several resistance determinants and then transfer them to Enterobacteriaceae and Pseudomonas spp.

\section{NDM Variants}

Since the first description of NDM-1, eight variants of this enzyme have been published (NDM-1 to -8) (Figure 4) and ten have been assigned (http://www.lahey.org). The first variant NDM-2 is a point mutation variant having a $\mathrm{C}$ to G substitution at position 82 resulting in an amino acid substitution of a proline to an alanine residue at position 28 (Pro $\rightarrow$ Ala) (Figure 4) [26]. Considering that this point mutation was located at the last amino acid of the peptide leader of the enzyme, MIC values of $\beta$-lactams including carbapenems showed no significant difference between NDM-1 and NDM- 2 producers. NDM- 2 has been identified in several A. baumannii strains $[20-22,26]$ but not yet in Enterobacteriaceae. The NDM-3 variant was described from an E. coli isolate and differs from NDM-1 by a single nucleotide change conferring a peptide sequence change at position 95 (Asp $\rightarrow$ Asn) that does not modify the hydrolytic activities of the enzyme (Figure 4) [11]. The NDM-4 variant differs from NDM-1 by a single amino acid substitution at position 154 $($ Met $\rightarrow$ Leu) (Figure 4). Kinetic data showed that this amino acid substitution is responsible for an increased hydrolytic activity of NDM- 4 compared to NDM- 1 toward cefalotin, ceftazidime, cefotaxime, imipenem, and meropenem, whereas cefepime was less hydrolyzed [72]. The NDM-5 variant shares the substitution at positions 154 (Met $\rightarrow$ Leu) with NDM-4, conferring enhanced hydrolytic activity against carbapenems 


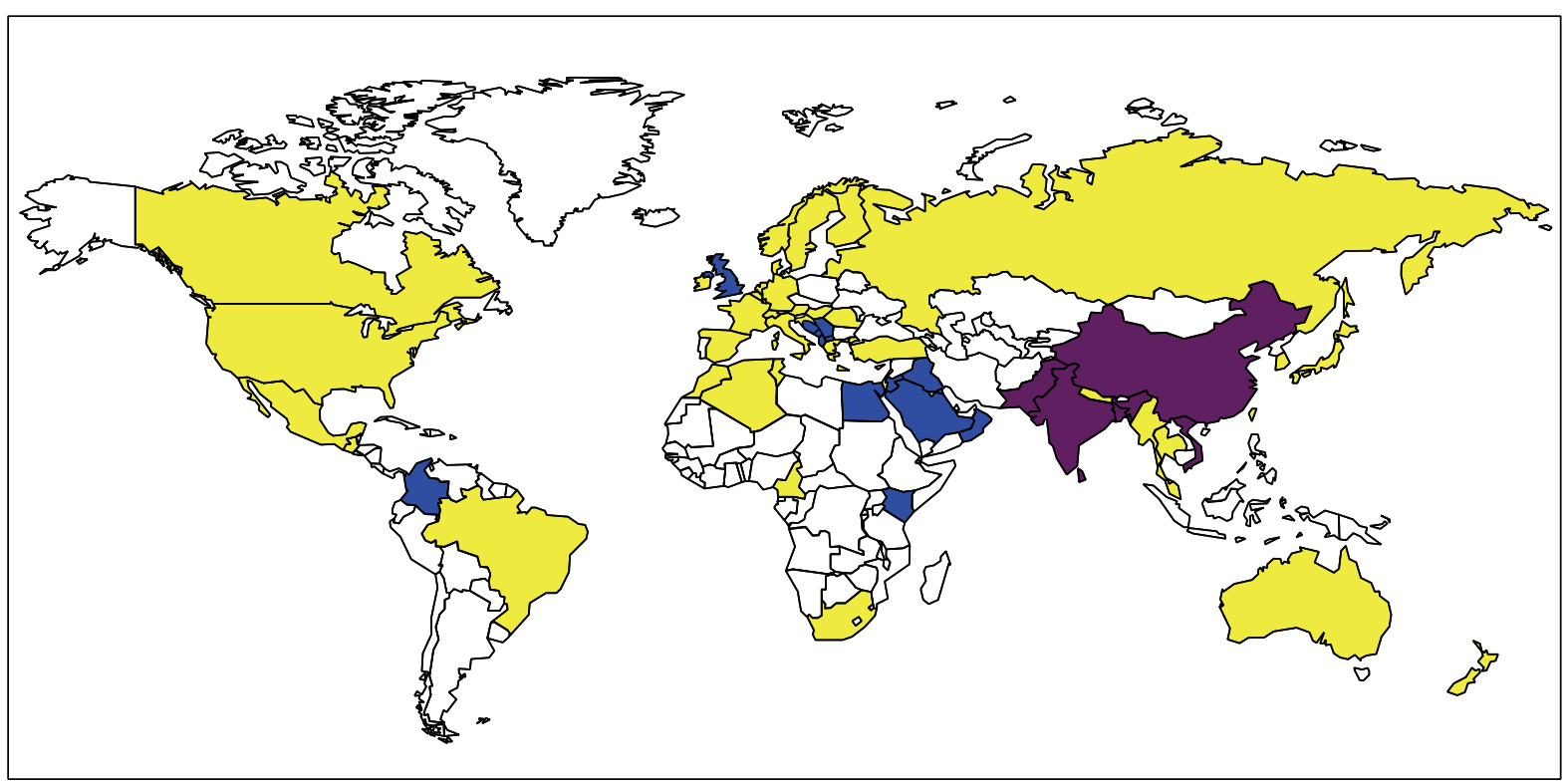

High prevalence of NDM producers (endemicity)

Outbreaks and interregional spread of NDM producers

Sporadic description of NDM producers

FIGURE 2: Geographical distribution of NDM producers.

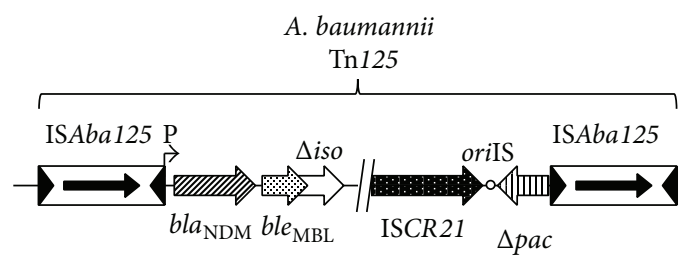

(a)

Enterobacteriaceae and P. aeruginosa

ISAba125
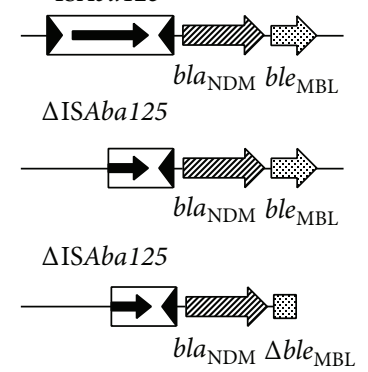

(b)

FIGURE 3: Schematic representation of $b l a_{\mathrm{NDM}^{-}}$-associated genetic structures identified among Gram-negative clinical isolates. (a) Structure found in A. baumannii, where the bla $a_{\mathrm{NDM}}$ gene is part of the composite transposon Tn125. (b) Structures found in Enterobacteriaceae and

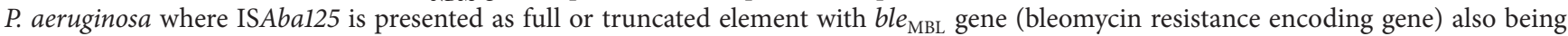
present as full or truncated gene. Genes and their corresponding transcription orientations are represented by horizontal arrows. oriIS of ISCR21 is indicated by a circle. The $b l a_{\mathrm{NDM}}$ promoter is indicated (P). IS, insertion sequence; gene names are abbreviated according to their corresponding proteins: $b l e_{\mathrm{MBL}}$, bleomycin resistance gene; $\Delta i s o$ for truncated phosphoribosylanthranilate isomerase; $\Delta p a c$ for truncated phospholipid acetyltransferase. 

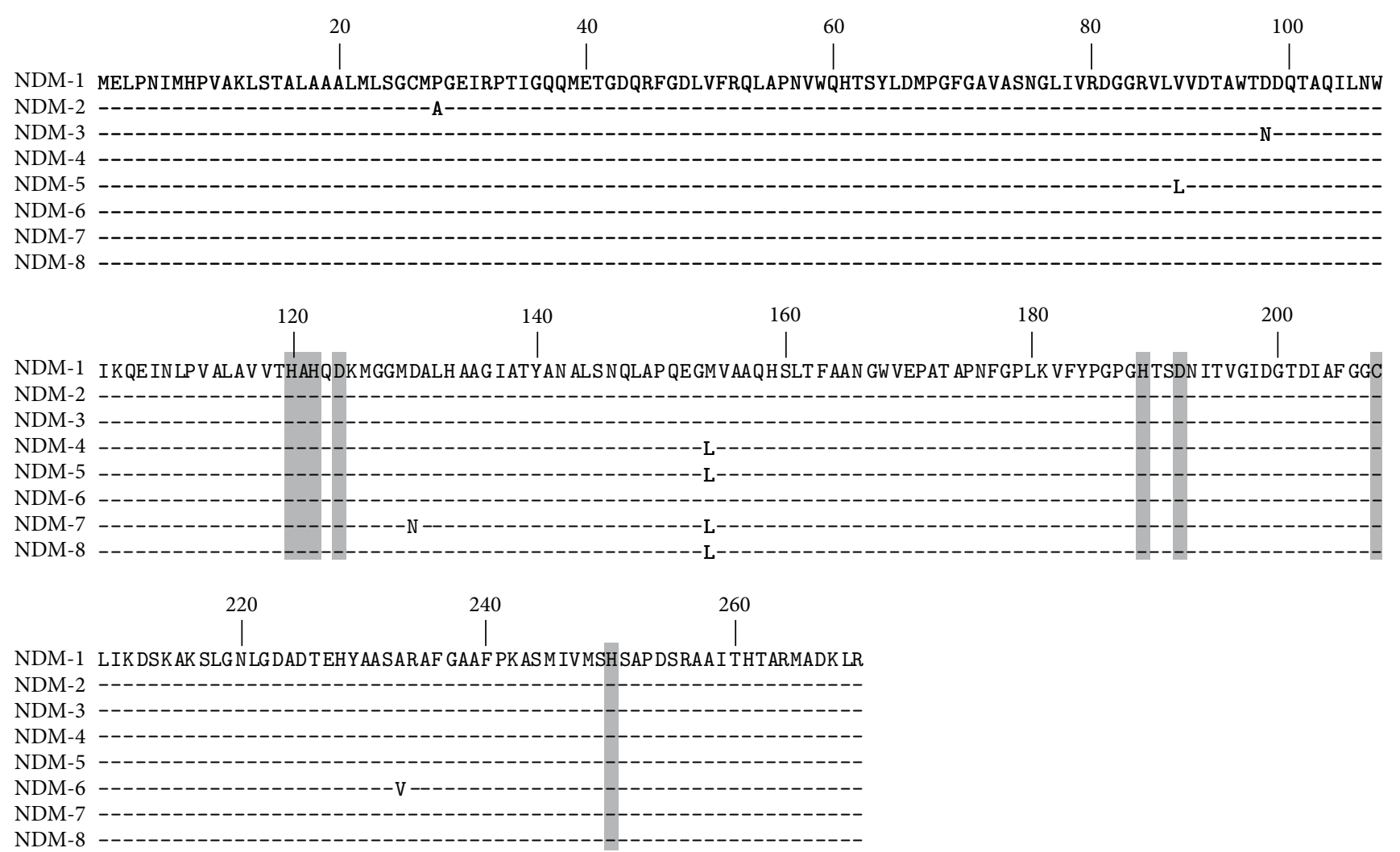

FIGURE 4: Alignment of the amino acid sequences of the eight reported NDM variants. Conserved residues of the active site of metallo$\beta$-lactamases are highlighted in gray. The bolded leucine in position 154 has been described to be responsible to an increased carbapenem hydrolysis.

and harbors a second amino acid substitution at position 88 (Val $\rightarrow$ Leu) (Figure 4) [73]. The NDM-6 variant differs from NDM-1 by a single amino acid substitution at position 233 (Ala $\rightarrow$ Val) leading to no obvious modification in the hydrolytic activity of the enzyme (Figure 4) [74]. The NDM7 variant was concomitantly described from an E. coli isolate recovered from a French patient who had travelled to Burma [75] and an E. coli isolate recovered from a Yemeni patient previously hospitalized at the Frankfurt University Hospital in Germany [76]. The $b l a_{\mathrm{NDM}-7}$ gene differs from $b l a_{\mathrm{NDM}-1}$ by two amino-acid substitutions at positions $388(\mathrm{G} \rightarrow \mathrm{A})$ and $460(\mathrm{~A} \rightarrow \mathrm{C})$ corresponding to amino acid substitutions at position 130 (Asp $\rightarrow$ Asn) and 154 (Met $\rightarrow$ Leu), respectively (Figure 4). The amino acid substitution at position 154 (Met $\rightarrow$ Leu) increases the hydrolysis activity of the enzyme [75, 76]. The amino acid sequence of the last published NDM variant, namely, NDM-8, has substitutions at positions 130 $($ Asp $\rightarrow$ Gly) and 154 (Met $\rightarrow$ Leu) compared with NDM-1 (Figure 4) [77]. This NDM variant possesses the amino acid substitution at position 154 (Met $\rightarrow$ Leu), but its critical impact on $\beta$-lactams hydrolysis has not been detailed.

\section{Identification of NDM Producers}

Detection of carbapenemase producers, including NDM producers, in clinical specimens is based currently on a preliminary analysis of susceptibility testing results. The US guidelines (CLSI) (updated in 2013) retained as breakpoints for Enterobacteriaceae susceptibility $(S) \leq 1$ and resistance $(R)>4 \mathrm{mg} / \mathrm{L}$ for imipenem and meropenem and $S \leq 0.5$ and $R>2 \mathrm{mg} / \mathrm{L}$ for ertapenem. The European guidelines (EUCAST) (updated in 2013) are slightly different and propose breakpoints for imipenem and meropenem as follows: susceptible $(S) \leq 2$ and resistant $(R)>8 \mathrm{mg} / \mathrm{L}$ and for ertapenem $S \leq 0.5$ and $R>1 \mathrm{mg} / \mathrm{L}$. Although some discrepancies might exist for several isolates depending on the reference used to interpret the antibiogram, MIC values of ertapenem are often higher than those of other carbapenems with NDM producers. Consequently, ertapenem would be the best molecule for suspecting most of the carbapenemase producers, including NDM producers, and constitutes good screening criteria. Notably, this greater sensitivity of ertapenem compared to the other carbapenems is counterbalanced by its lower specificity. Of note, susceptibility to carbapenems is observed for some NDM producers and additional tests for carbapenemase detection are needed to detect them accurately.

7.1. Detection of a Carbapenemase Activity. One of the commonly used techniques is the modified Hodge test (MHT), which has been used for years. Unfortunately, the MHT has been proved to lack sensitivity (50\%) for detecting NDM1 producers. Of note, $\mathrm{ZnSO}_{4}(100 \mu \mathrm{g} / \mathrm{mL})$ supplementation in the culture medium significantly increases the sensitivity 
to $85.7 \%$ [78]. However, this test has a low specificity with Enterobacter spp. often overexpressing their chromosomal cephalosporinase [79]. In addition, results of the MHT are obtained at least $72 \mathrm{~h}$ after the bacterial identification.

UV spectrophotometry analysis of carbapenem hydrolysis has been developed to detect carbapenem hydrolysis. This method is based on the detection of the decrease of imipenem absorbance with crude extracts of bacterial enzymes. Crude extracts can be obtained from an overnight culture of the tested strain after mechanical lysis. This UV spectrophotometry-based technique is cheap and has a $100 \%$ sensitivity and a $98.5 \%$ specificity for detecting carbapenemase activity [80]. However, it is time-consuming and requires trained microbiologists and expensive equipment.

Analysis of carbapenem hydrolysis by using the MALDITOF technology has been shown to be a useful technique to detect carbapenemase production in a few hours. This technique was based on detection of a carbapenem (imipenem, meropenem, or ertapenem) spectrum and of its main derivatives resulting from carbapenem hydrolysis. After 3 to 4 hours of incubation of the tested isolate with a carbapenem, the bacteria were pelleted by centrifugation and the supernatant containing the carbapenem and its metabolites was tested by MALDI-TOF mass spectrophotometry. Disappearance of the peak corresponding to the native carbapenem and appearance of peak(s) corresponding to the metabolite(s) resulting on the carbapenem hydrolysis sign a carbapenemase activity [81-85]. This test has excellent sensitivity and specificity. However, it again requires trained microbiologists and expensive equipment.

The most promising technique is the rapid Carba NP test. It is based on the detection of the hydrolysis of imipenem by a color change of a $\mathrm{pH}$ indicator (Figure 5 ). This test is $100 \%$ sensitive and $100 \%$ specific for the detection of any type of carbapemenase produced by Enterobacteriaceae including NDM producers [86-88]. The Carba NP test has been also validated for the detection of most carbapenemaseproducing Pseudomonas spp., including all NDM producers [89]. A second version of the Carba NP test (the Carba NP test II) has been developed to rapidly differentiate between the diverse carbapenemase types found in Enterobacteriaceae and $P$. aeruginosa. This Carba NP test II combines the inhibition properties of EDTA with the high efficiency of the Carba NP test for identification of any type of MBL producer, including all NDM producers [90]. Recently, the Carba NP test has been evaluated to detect carbapenemase-producing Enterobacteriaceae $(n=193)$ directly from spiked blood cultures. The proposed strategy allows detection of all NDM producers $(n=33)$ in less than 5 hours, with sensitivity and specificity of $100 \%$, respectively [91]. This test has excellent sensitivity and specificity. However, it requires homemade reagents that are not yet commercially available.

7.2. Phenotypic Detection of Metallo- $\beta$-Lactamase (MBL) Producing Isolates. Detection methods based on the inhibitory properties of several divalent ions chelators (e.g., EDTA and dipicolinic acid) may identify MBL producers. A diskdiffusion test based on the detection of a synergy between a carbapenem-containing disk (imipenem or meropenem) and a disk containing an MBL inhibitor (EDTA or mercaptopropionic acid or dipicolinic acid) has been proposed [92].

A combined disk technique using a carbapenem disk and the same carbapenem disk supplemented with EDTA $(10 \mu \mathrm{L}$ of a $0.1 \mathrm{M}$ solution at $\mathrm{pH} 8)$ has been also proposed [93]. Using this test, a $5 \mathrm{~mm}$ increase of the inhibition diameter around the disk containing imipenem plus EDTA compared to imipenem alone likely indicates the production of a MBL. However, those two phenotypic methods are timeconsuming and false-negative results often arise, in particular when low level of resistance is observed [93].

Among those phenotypic methods, the Etest MBL strip, a two-sided strip containing gradients of imipenem alone on one side and imipenem supplemented with EDTA on the other side, is also commonly used for the detection of MBL producers. Using this test, at least three doubling dilutions of the MIC in the presence of EDTA are considered as a positive result [94]. However, several NDM-producing isolates exhibit low MIC of carbapenems, leading to not interpretable results using the Etest MBL strip.

7.3. Molecular Detection of NDM Producers. All the previous techniques can detect the carbapenemase production and, in some cases more precisely, production of an MBL, but none of them is able to specifically identify an NDM enzyme or its corresponding gene. Therefore a number of genotypic approaches have been reported, based on PCR techniques, including real-time PCR methods able to detect $b l a_{\mathrm{NDM}}$-positive isolates directly from clinical samples [95, 96]. Those methods, however, have the disadvantage to be unable to identify any novel carbapenemase gene and are quite expensive. Commercial DNA microarray methods are marketed and increase the convenience of those tests [97]. Although they cannot overcome general limitations of genotypic techniques those DNA microarrays are able to identify the presence of carbapenemase and the main extended-spectrum $\beta$-lactamase and acquired cephalosporinases genes. Accordingly, this technique is more adapted for an epidemiological purpose in order to control an outbreak. Finally, molecular amplification of the $b l a_{\mathrm{NDM}}$ gene followed by sequencing is needed to identify the exact nature of the NDM variant.

\section{Detection of Infected and Colonized Patients}

Since the prevention of dissemination of carbapenemase producers partially relies on an early and accurate detection of carriers, recommendations for the screening of colonized patients have been introduced in several countries. Commonly, "at-risk" patients, meaning those being colonized with carbapenemase producers, are patients transferred from a foreign hospital and those hospitalized in intensive-care units, in transplantation units, and immunocompromised patients.

Since the intestinal flora is the main reservoir of Enterobacteriaceae, rectal swabs and stools are the most suitable clinical samples for performing this screening. These specimens may be plated on screening medium, either directly 
<smiles>[R]C1=C2CCN(C(C(=O)O)C2)C1C(=O)O</smiles>

Carbapenem Imipenem
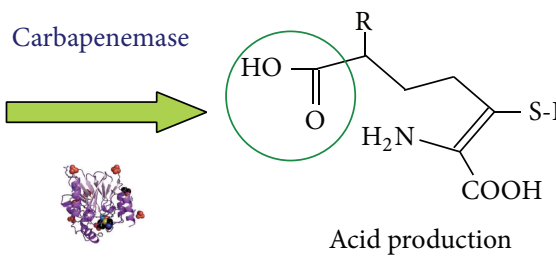

Acid production

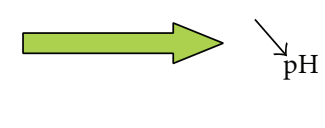

Colorimetric detection

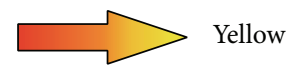

(a)

No carbapenemase

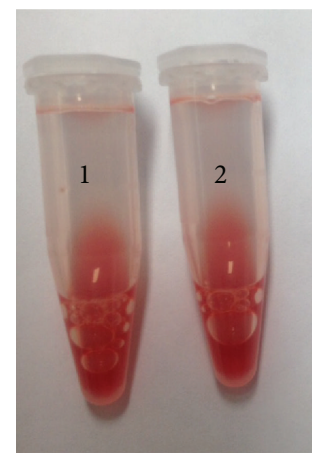

Carbapenemase production

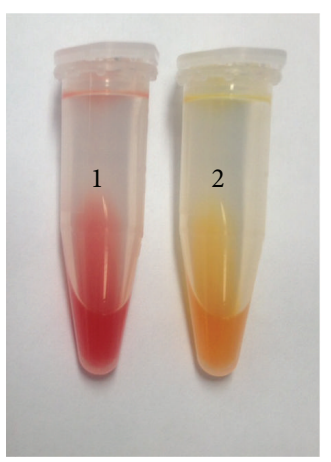

(1) Revealing solution

(internal negative control)

(2) Revealing solution + imipenem

(b)

Figure 5: Principle (a) and interpretation (b) of the Carba NP test recently developed for the rapid identification of carbapenemase producers among Enterobacteriaceae and Pseudomonas spp.

or after an enrichment step in broth containing imipenem $0.5-1 \mu \mathrm{g} / \mathrm{mL}$ or ertapenem $0.5 \mu \mathrm{g} / \mathrm{mL}[98,99]$. In outbreak situations, this enrichment step might increase the sensitivity of the screening and consequently reduce the number of potential false-negative results by increasing the inoculum of the targeted strain. On the opposite, its disadvantage is the induced delay $(12-24 \mathrm{~h})$ needed to confirm or reject carbapenemase detection. Although the efficiency of this enrichment step has not been evaluated for NDM producers, it has already been shown to improve the detection of KPC producers.

Regardless of the enrichment step, the specimens have to be plated on selective media. For that purpose, several screening media have been evaluated and compared to the screening of carriers of NDM producers. One of the first tested medium was the ChromID ESBL culture medium (bioMérieux) containing cefpodoxime used as a selector and which is routinely used to screen ESBL producers. Since NDM enzymes have a broad-spectrum activity, they hydrolyze not only carbapenems but also expanded-spectrum cephalosporins very efficiently. Therefore, detection of NDM-producing isolates using ChromID ESBL (aimed do detect ESBL producers) is possible but with a low specificity since the selective agent is a cephalosporin and not a specific carbapenemase substrate (e.g., a carbapenem). Several media supplemented with a carbapenem have been developed and marketed for the screening of carbapenemase producers. The first screening medium targeting KPC producers was the CHROMagar KPC medium that contains meropenem (CHROMagar, Paris, France) [100]. Using this medium, carbapenem-resistant bacteria are well detected when they exhibit relatively high-level resistance to carbapenems. Its main disadvantage remains in its lack of sensitivity, since it does not detect carbapenemase producers with low-level carbapenem resistance. Indeed, although NDM producers have often high level resistance to carbapenems, several isolates that exhibited MICs comprised between 0.5 and $1 \mu \mathrm{g} / \mathrm{mL}$, making their detection difficult on screening media containing high concentration of carbapenems [78, 93, 101]. Colorex KPC (E\&O laboratories, Bonnybridge, UK), another screening medium for carbapenemase producers, also contains meropenem. Since the content of this medium is reported to be identical to that of CHROMagar KPC, only NDM producers with highlevel resistance to carbapenems may be detected, leading to an accurate detection of $57 \%$ to $64 \%$ of NDM-producing Enterobacteriaceae using this medium [55, 102]. A third commercially available screening medium also contains a carbapenem (CRE Brilliance, Thermo Fisher Scientific, UK). Depending on the study, sensitivities for detection of patients colonized with NDM producers were reported to be $63 \%$ 
to $85 \%$ using this medium $[102,103]$. Another screening medium also containing a carbapenem is the ChromID CARBA (bioMérieux, La Balmes-les-Grottes, France). This commercially available medium has been reported to be more sensitive $(87.5 \%$ to $94 \%)$ than the others for the detection of NDM-producing Enterobacteriaceae [55, 102, 103]. Finally, a homemade screening medium containing ertapenem, cloxacillin, and zinc, namely, the SUPERCARBA medium, has an excellent sensitivity and specificity for the detection of carbapenemase producers, including NDM producers. The zinc supplementation and the low ertapenem concentration allow the efficient detection of all NDM producers regardless of their level of resistance to carbapenems $[101,104]$. Consequently, using the SUPERCARBA medium and performing the Carba NP test on isolated colonies might be proposed as the recommended strategy for screening of carbapenemase producers [105].

In order to avoid the additional $24 \mathrm{~h}$ to $48 \mathrm{~h}$ before the carriage status of the patient can be established using those screening media an in-house quantitative real-time PCR assay using the TaqMan chemistry has been developed to detect the NDM-encoding genes directly from spiked stool samples. The bacterial extraction from stool samples was performed manually or adapted to a fully automated extraction system. This assay was found to be $100 \%$ specific and sensitive with detection limits reproducible below $1 \times$ $10^{1} \mathrm{CFU} / 100 \mathrm{mg}$ of feces [95]. However, this technology remains expensive and is thus considered to be a valuable tool in the follow-up of an outbreak and cohorting of colonized patients.

\section{Conclusion}

The rapid diffusion of NDM producers is of particular concern since this now corresponds to a worldwide-located outbreak. Additional knowledge in relation to the driven forces behind the spread of those multidrug-resistant isolates is now required, in order to better understand the dynamics of the NDM producers.

Among the most important features of NDM producers, one may retain that those NDM producers are not only nosocomial enterobacterial pathogens, but also communityacquired Enterobacteriaceae or other Gram-negative species, such as A. baumannii. Such diffusion pattern of multidrug resistance is unique for NDM producers and not observed currently for none of the producers of other types of carbapenemases (OXA-48, KPC, IMP...).

The reservoir of those NDM producers is mainly located in Southeast Asia where the rate of carriers is estimated to be ca. $20 \%$. However, it is difficult to predict the number of tourists in India, Pakistan, and Bangladesh (more than 10 million in 2012) that will bring back those NDM producers at a carrier stage in stools. The size of that reservoir may explain the rapidity of the dissemination of NDM producers worldwide. Accordingly, NDM producers are now on the top list of carbapenemase producers in European countries such as the UK and even in France.

Due to the population demography of the NDM reservoirs coupled with the difficulties to implement hygiene measures or an efficient antibiotic stewardship program in those countries, the outbreak will not stop spontaneously. On the opposite, we will see an acceleration of the NDM producers spread worldwide. Hopefully the rate of NDM producers will not reach those currently observed for ESBL producers (20-80\% worldwide). The spread of ESBL producers is an important driving force for usage of carbapenems that will enhance selection of carbapenemase producers. The only significant action we may actually take currently in Western countries which are not endemic for carbapenemase producers is to sustain the wide usage of rapid detection techniques now available and the extended screening of potential carriers at least in hospitals.

It is likely that novel antibiotic molecules such as the combination of aztreonam and avibactam may be launched in a near future and may bring some therapeutical openings. However there is an urgent need to finance novel research programs for discovering novel anti-Gram negatives molecules and to implement worldwide-located surveillance network of multidrug antibiotic resistance with sentinel labs.

\section{Conflict of Interests}

The authors declare that there is no conflict of interests regarding the publication of this paper.

\section{References}

[1] J. F. Wang and K. C. Chou, "Metallo- $\beta$-lactamases: structural features, antibiotic recognition, inhibition, and inhibitor design," Current Topics in Medicinal Chemistry, vol. 13, no. 10, pp. 1242-1253, 2013.

[2] A. Yoshizumi, Y. Ishii, D. M. Livermore et al., "Efficacies of calcium-EDTA in combination with imipenem in a murine model of sepsis caused by Escherichia coli with NDM-1 $\beta$ lactamase," Journal of Infection and Chemotherapy, vol. 19, no. 5, pp. 992-995, 2013.

[3] A. Pal and A. Tripathi, "An in silico approach for understanding the molecular evolution of clinically important metallo- $\beta$ lactamases," Infection, Genetics and Evolution, vol. 20, pp. 39-47, 2013.

[4] A. Barguigua, F. El Otmani, F. Lakbakbi El Yaagoubi, M. Talmi, K. Zerouali, and M. Timinouni, "First report of a Klebsiella pneumoniae strain coproducing NDM-1, VIM-1 and OXA48 carbapenemases isolated in Morocco," Acta Pathologica, Mrobiologica et Immunologica Scandinavica, vol. 121, no. 7, pp. 675-677, 2013.

[5] M. Castanheira, L. M. Deshpande, D. Mathai, J. M. Bell, R. N. Jones, and R. E. Mendes, "Early dissemination of NDM-1- and OXA-181-producing Enterobacteriaceae in Indian hospitals: report from the SENTRY Antimicrobial Surveillance Program, 2006-2007," Antimicrobial Agents and Chemotherapy, vol. 55, no. 3, pp. 1274-1278, 2011.

[6] M. Dolejska, L. Villa, L. Poirel, P. Nordmann, and A. Carattoli, "Complete sequencing of an IncHIl plasmid encoding the carbapenemase NDM-1, the ArmA 16S RNA methylase and a resistance-nodulation-cell division/multidrug efflux pump," Journal of Antimicrobial Chemotherapy, vol. 68, no. 1, pp. 34-39, 2013. 
[7] L. Poirel, L. Dortet, S. Bernabeu, and P. Nordmann, "Genetic features of $b l a_{\mathrm{NDM}-1}$-positive Enterobacteriaceae," Antimicrobial Agents and Chemotherapy, vol. 55, no. 11, pp. 5403-5407, 2011.

[8] L. Poire, A. Ros, A. Carricajo et al., "Extremely drug-resistant Citrobacter freundii isolate producing NDM-1 and other carbapenemases identified in a patient returning from India," Antimicrobial Agents and Chemotherapy, vol. 55, no. 1, pp. 447448, 2011.

[9] O. Samuelsen, U. Naseer, N. Karah et al., "Identification of Enterobacteriaceae isolates with OXA-48 and coproduction of OXA-181 and NDM-1 in Norway," Journal of Antimicrobial Chemotherapy, vol. 68, no. 7, pp. 1682-1685, 2013.

[10] M. E. Falagas, D. E. Karageorgopoulos, and P. Nordmann, "Therapeutic options for infections with Enterobacteriaceae producing carbapenem-hydrolyzing enzymes," Future Microbiology, vol. 6, no. 6, pp. 653-666, 2011.

[11] B. A. Rogers, H. E. Sidjabat, A. Silvey et al., "Treatment options for New Delhi metallo- $\beta$-lactamase-harboring Enterobacteriaceae," Microbial Drug Resistance, vol. 19, no. 2, pp. 100-103, 2013.

[12] B. Berçot, L. Poirel, L. Dortet, and P. Nordmann, "In vitro evaluation of antibiotic synergy for NDM-1-producing Enterobacteriaceae," Journal of Antimicrobial Chemotherapy, vol. 66, no. 10, pp. 2295-2297, 2011.

[13] D. M. Livermore, S. Mushtaq, M. Warner et al., "Activities of NXL104 combinations with ceftazidime and aztreonam against carbapenemase-producing Enterobacteriaceae," Antimicrobial Agents and Chemotherapy, vol. 55, no. 1, pp. 390-394, 2011.

[14] S. Shakil, E. I. Azhar, S. Tabrez et al., "New Delhi metallo- $\beta$ lactamase (NDM-1): an update," Journal of Chemotherapy, vol. 23, no. 5, pp. 263-265, 2011.

[15] P. Bogaerts, R. Rezende de Castro, S. Roisin et al., "Emergence of NDM-1-producing Acinetobacter baumannii in Belgium," Journal of Antimicrobial Chemotherapy, vol. 67, no. 6, pp. 15521553, 2012.

[16] R. A. Bonnin, T. Naas, L. Poirel, and P. Nordmann, "Phenotypic, biochemical, and molecular techniques for detection of metallo- $\beta$-lactamase NDM in Acinetobacter baumannii," Journal of Clinical Microbiology, vol. 50, no. 4, pp. 1419-1421, 2012.

[17] A. Boulanger, T. Naas, N. Fortineau, S. Figueiredo, and P. Nordmann, "NDM-1-producing Acinetobacter baumannii from Algeria," Antimicrobial Agents and Chemotherapy, vol. 56, no. 4, pp. 2214-2215, 2012.

[18] Y. Chen, Y. Cui, F. Pu et al., "Draft genome sequence of an Acinetobacter genomic species 3 strain harboring a $b l a_{\mathrm{NDM}-1}$ gene," Journal of Bacteriology, vol. 194, no. 1, pp. 204-205, 2012.

[19] Y. Chen, Z. Zhou, Y. Jiang, and Y. Yu, "Emergence of NDM1-producing Acinetobacter baumannii in China," Journal of Antimicrobial Chemotherapy, vol. 66, no. 6, pp. 1255-1259, 2011.

[20] P. Espinal, G. Fugazza, Y. López et al., "Dissemination of an NDM-2-producing Acinetobacter baumannii clone in an Israeli Rehabilitation Center," Antimicrobial Agents and Chemotherapy, vol. 55, no. 11, pp. 5396-5398, 2011.

[21] P. Espinal, L. Poirel, Y. Carmeli et al., "Spread of NDM-2producing Acinetobacter baumannii in the Middle East," Journal of Antimicrobial Chemotherapy, vol. 68, no. 8, pp. 1928-1930, 2013.

[22] A. Ghazawi, Á. Sonnevend, R. A. Bonnin et al., "NDM2 carbapenemase-producing Acinetobacter baumannii in the United Arab Emirates," Clinical Microbiology and Infection, vol. 18, no. 2, pp. E34-E36, 2012.
[23] A. M. Hammerum, A. R. Larsen, F. Hansen et al., "Patients transferred from Libya to Denmark carried OXA-48-producing Klebsiella pneumoniae, NDM-1-producing Acinetobacter baumannii and meticillin-resistant Staphylococcus aureus," International Journal of Antimicrobial Agents, vol. 40, no. 2, pp. 191-192, 2012.

[24] J. Hrabák, M. Štolbová, V. Študentová, M. Fridrichová, E. Chudáčková, and H. Zemlickova, "NDM-1 producing Acinetobacter baumannii isolated from a patient repatriated to the Czech Republic from Egypt, July 2011," Eurosurveillance, vol. 17, no. 7, 2012.

[25] H. Hu, Y. Hu, Y. Pan et al., "Novel plasmid and its variant harboring both a $b l a_{\mathrm{NDM}-1}$ gene and type IV secretion system in clinical isolates of Acinetobacter lwoffi," Antimicrobial Agents and Chemotherapy, vol. 56, no. 4, pp. 1698-1702, 2012.

[26] M. Kaase, P. Nordmann, T. A. Wichelhaus, S. G. Gatermann, R. A. Bonnin, and L. Poirel, "NDM-2 carbapenemase in Acinetobacter baumannii from Egypt," Journal of Antimicrobial Chemotherapy, vol. 66, no. 6, pp. 1260-1262, 2011.

[27] Y. Nakazawa, R. Ii, T. Tamura et al., "A case of NDM-1producing Acinetobacter baumannii transferred from India to Japan," Journal of Infection and Chemotherapy, vol. 19, no. 2, pp. 330-332, 2013.

[28] A. Nemec and L. Krizova, "Carbapenem-resistant Acinetobacter baumannii carrying the NDM-1 gene, Czech Republic, 2011," Eurosurveillance, vol. 17, no. 11, 2012.

[29] Y. Sun, Y. Song, H. Song et al., "Complete genome sequence of an Acinetobacter strain harboring the NDM-1 gene," Genome Announcements, vol. 1, no. 2, Article ID e0002312, 2013.

[30] J. Yang, Y. Chen, X. Jia et al., "Dissemination and characterization of NDM-1-producing Acinetobacter pittii in an intensive care unit in China," Clinical Microbiology and Infection, vol. 18, no. 12, pp. E506-E513, 2012.

[31] C. Zhang, S. Qiu, Y. Wang et al., "Higher isolation of NDM1 producing Acinetobacter baumannii from the sewage of the hospitals in Beijing," PLoS ONE, vol. 8, no. 6, Article ID e64857, 2013.

[32] Z. Zhou, R. Guan, Y. Yang et al., "Identification of New Delhi metallo- $\beta$-lactamase gene (NDM-1) from a clinical isolate of Acinetobacter junii in China," Canadian Journal of Microbiology, vol. 58, no. 1, pp. 112-115, 2012.

[33] C. Flateau, F. Janvier, H. Delacour et al., "Recurrent pyelonephritis due to NDM- 1 metallo- $\beta$-lactamase producing Pseudomonas aeruginosa in a patient returning from Serbia, France, 2012," Eurosurveillance, vol. 17, no. 45, 2012.

[34] B. Jovcic, Z. Lepsanovic, V. Suljagic et al., "Emergence of NDM1 metallo- $\beta$-lactamase in Pseudomonas aeruginosa clinical isolates from Serbia," Antimicrobial Agents and Chemotherapy, vol. 55, no. 8, pp. 3929-3931, 2011.

[35] R. A. Bonnin, L. Poirel, A. Carattoli, and P. Nordmann, "Characterization of an IncFII plasmid encoding NDM-1 from Escherichia coli ST131," PLoS ONE, vol. 7, no. 4, Article ID e34752, 2012.

[36] A. Hishinuma, A. Yoshida, H. Suzuki, K. Okuzumi, and T. Ishida, "Complete sequencing of an IncFII NDM-1 plasmid in Klebsiella pneumoniae shows structural features shared with other multidrug resistance plasmids," Journal of Antimicrobial Chemotherapy, vol. 68, no. 10, pp. 2415-2417, 2013.

[37] P. Mc Gann, J. Hang, R. J. Clifford et al., "Complete sequence of a novel 178-kilobase plasmid carrying bla $_{\mathrm{NDM}-1}$ in a Providencia stuartii strain isolated in Afghanistan," Antimicrobial Agents and Chemotherapy, vol. 56, no. 4, pp. 1673-1679, 2012. 
[38] T. Sekizuka, M. Matsui, K. Yamane et al., "Complete sequencing of the $b l a_{\mathrm{NDM}-1}$-positive IncA/C plasmid from Escherichia coli ST38 isolate suggests a possible origin from plant pathogens," PLoS ONE, vol. 6, no. 9, Article ID e25334, 2011.

[39] T. Yamamoto, T. Takano, T. Fusegawa et al., "Electron microscopic structures, serum resistance, and plasmid restructuring of New Delhi metallo- $\beta$-lactamase-1 (NDM-1)-producing ST42 Klebsiella pneumoniae emerging in Japan," Journal of Infection and Chemotherapy, vol. 19, no. 1, pp. 118-127, 2013.

[40] K. Fuursted, L. Schøler, F. Hansen et al., "Virulence of a Klebsiella pneumoniae strain carrying the new delhi metallobeta-lactamase-1 (ndm-1)," Microbes and Infection, vol. 14, no. 2, pp. 155-158, 2012.

[41] G. Peirano, G. L. Mulvey, G. D. Armstrong, and J. D. Pitout, "Virulence potential and adherence properties of Escherichia coli that produce CTX-M and NDM $\beta$-lactamases," Journal of Medical Microbiology, vol. 62, part 4, pp. 525-530, 2013.

[42] G. Peirano, P. C. Schreckenberger, and J. D. D. Pitout, "Characteristics of NDM-1-producing Escherichia coli isolates that belong to the successful and virulent clone ST131," Antimicrobial Agents and Chemotherapy, vol. 55, no. 6, pp. 2986-2988, 2011.

[43] F. Cabanes, J. Lemant, S. Picot et al., "Emergence of Klebsiella pneumoniae and Salmonella metallo- $\beta$-lactamase (NDM-1) producers on Reunion island," Journal of Clinical Microbiology, vol. 50, no. 11, p. 3812, 2012.

[44] J. Fischer, S. Schmoger, S. Jahn, R. Helmuth, and B. Guerra, "NDM-1 carbapenemase-producing Salmonella enterica subsp. enterica serovar Corvallis isolated from a wild bird in Germany," Journal of Antimicrobial Chemotherapy, vol. 68, no. 12, pp. 29542956, 2013.

[45] P. Savard, R. Gopinath, W. Zhu et al., "First NDM-positive Salmonella sp. strain identified in the United States," Antimicrobial Agents and Chemotherapy, vol. 55, no. 12, pp. 5957-5958, 2011.

[46] E. Darley, J. Weeks, L. Jones et al., "NDM-1 polymicrobial infections including Vibrio cholerae," The Lancet, vol. 380, no. 9850, p. 1358, 2012.

[47] T. R. Walsh, J. Weeks, D. M. Livermore, and M. A. Toleman, "Dissemination of NDM-1 positive bacteria in the New Delhi environment and its implications for human health: an environmental point prevalence study," The Lancet Infectious Diseases, vol. 11, no. 5, pp. 355-362, 2011.

[48] D. Yong, M. A. Toleman, C. G. Giske et al., "Characterization of a new metallo- $\beta$-lactamase gene, bla NDM-1, and a novel erythromycin esterase gene carried on a unique genetic structure in Klebsiella pneumoniae sequence type 14 from India," Antimicrobial Agents and Chemotherapy, vol. 53, no. 12, pp. 5046-5054, 2009.

[49] K. K. Kumarasamy, M. A. Toleman, T. R. Walsh et al., "Emergence of a new antibiotic resistance mechanism in India, Pakistan, and the UK: a molecular, biological, and epidemiological study," The Lancet Infectious Diseases, vol. 10, no. 9, pp. 597-602, 2010.

[50] M. J. Struelens, D. L. Monnet, A. P. Magiorakos, F. S. O’Connor, J. Giesecke, and European NDM-1 Survey Participants, "New Delhi metallo-beta-lactamase 1-producing Enterobacteriaceae: emergence and response in Europe," Eurosurveillance, vol. 15, no. 46, 2010.

[51] C. Lascols, M. Hackel, S. H. Marshall et al., "Increasing prevalence and dissemination of NDM- 1 metallo- $\beta$-lactamase in India: data from the SMART study (2009)," Journal of Antimicrobial Chemotherapy, vol. 66, no. 9, pp. 1992-1997, 2011.
[52] P. Deshpande, C. Rodrigues, A. Shetty, F. Kapadia, A. Hedge, and R. Soman, "New Delhi Metallo- $\beta$ lactamase (NDM-1) in enterobacteriaceae: treatment options with carbapenems compromised," Journal of Association of Physicians of India, vol. 58, no. 3, pp. 147-149, 2010.

[53] P. Deshpande, A. Shetty, F. Kapadia, A. Hedge, R. Soman, and C. Rodrigues, "New Delhi metallo 1: have carbapenems met their doom?” Clinical Infectious Diseases, vol. 51, no. 10, p. 1222, 2010.

[54] R. Bharadwaj, S. Joshi, V. Dohe, V. Gaikwad, G. Kulkarni, and Y. Shouche, "Prevalence of New Delhi metallo- $\beta$-lactamase (NDM-1)-positive bacteria in a tertiary care centre in Pune, India," International Journal of Antimicrobial Agents, vol. 39, no. 3, pp. 265-266, 2012.

[55] J. D. Perry, S. H. Naqvi, I. A. Mirza et al., "Prevalence of faecal carriage of Enterobacteriaceae with NDM-1 carbapenemase at military hospitals in Pakistan, and evaluation of two chromogenic media," Journal of Antimicrobial Chemotherapy, vol. 66, no. 10, pp. 2288-2294, 2011.

[56] A. Gecaj-Gashi, A. Hasani, B. Bruqi, and G. Mulliqi-Osmani, "Balkan NDM-1: escape or transplant?" The Lancet Infectious Diseases, vol. 11, no. 8, p. 586, 2011.

[57] D. M. Livermore, T. R. Walsh, M. Toleman, and N. Woodford, "Balkan NDM-1: escape or transplant?" The Lancet Infectious Diseases, vol. 11, no. 3, p. 164, 2011.

[58] T. Halaby, A. E. Reuland, N. Al Naiemi et al., "A case of New Delhi metallo- $\beta$-lactamase 1 (NDM-1)-producing Klebsiella pneumoniae with putative secondary transmission from the Balkan region in the Netherlands," Antimicrobial Agents and Chemotherapy, vol. 56, no. 5, pp. 2790-2791, 2012.

[59] A. Mazzariol, Z. Bošnjak, P. Ballarini et al., "NDM-1-producing Klebsiella pneumoniae, Croatia," Emerging Infectious Diseases, vol. 18, no. 3, pp. 532-534, 2012.

[60] L. Poirel, E. Lagrutta, P. Taylor, J. Pham, and P. Nordmann, "Emergence of metallo- $\beta$-lactamase NDM-1-producing multidrug-resistant Escherichia coli in Australia," Antimicrobial Agents and Chemotherapy, vol. 54, no. 11, pp. 4914-4916, 2010.

[61] G. Zarfel, M. Hoenigl, B. Würstl et al., "Emergence of carbapenem-resistant Enterobacteriaceae in Austria, 20012010," Clinical Microbiology and Infection, vol. 17, no. 11, pp. E5E8, 2011.

[62] H. M. Zowawi, H. H. Balkhy, T. R. Walsh, and D. L. Paterson, “ $\beta$ lactamase production in key gram-negative pathogen isolates from the Arabian peninsula," Clinical Microbiology Reviews, vol. 26, no. 3, pp. 361-380, 2013.

[63] A. Sonnevend, A. Al Baloushi, A. Ghazawi et al., "Emergence and spread of NDM-1 producer Enterobacteriaceae with contribution of IncX3 plasmids in the United Arab Emirates," Journal of Medical Microbiology, vol. 62, part 7, pp. 1044-1050, 2013.

[64] L. Poirel, Z. Al Maskari, F. Al Rashdi, S. Bernabeu, and P. Nordmann, "NDM-1-producing Klebsiella pneumoniae isolated in the Sultanate of Oman," Journal of Antimicrobial Chemotherapy, vol. 66, no. 2, pp. 304-306, 2011.

[65] W. Jamal, V. O. Rotimi, M. J. Albert, F. Khodakhast, E. E. Udo, and L. Poirel, "Emergence of nosocomial New Delhi metallo$\beta$-lactamase-1 (NDM-1)-producing Klebsiella pneumoniae in patients admitted to a tertiary care hospital in Kuwait," International Journal of Antimicrobial Agents, vol. 39, no. 2, pp. 183-184, 2012.

[66] L. Dortet, L. Poirel, F. Al Yaqoubi, and P. Nordmann, "NDM1, OXA-48 and OXA-181 carbapenemase-producing Enterobacteriaceae in Sultanate of Oman," Clinical Microbiology and Infection, vol. 18, no. 5, pp. E144-E148, 2012. 
[67] A. Shibl, M. Al-Agamy, Z. Memish, A. Senok, S. A. Khader, and A. Assiri, "The emergence of OXA-48- and NDM-1-positive Klebsiella pneumoniae in Riyadh, Saudi Arabia," International Journal of Infectious Diseases, vol. 17, no. 12, pp. el130-e1133, 2013.

[68] P. L. Ho, W. U. Lo, M. K. Yeung et al., "Complete sequencing of pNDM-HK encoding NDM-1 carbapenemase from a multidrug-resistant Escherichia coli strain isolated in Hong Kong," PLoS ONE, vol. 6, no. 3, Article ID e17989, 2011.

[69] L. Dortet, P. Nordmann, and L. Poirel, "Association of the emerging carbapenemase NDM-1 with a bleomycin resistance protein in Enterobacteriaceae and Acinetobacter baumannii," Antimicrobial Agents and Chemotherapy, vol. 56, no. 4, pp. 16931697, 2012.

[70] Y. Pfeifer, W. Witte, M. Holfelder, J. Busch, P. Nordmann, and L. Poirel, "NDM-1-producing Escherichia coli in Germany," Antimicrobial Agents and Chemotherapy, vol. 55, no. 3, pp. 13181319, 2011.

[71] L. Poirel, R. A. Bonnin, A. Boulanger, J. Schrenzel, M. Kaase, and P. Nordmann, "Tn125-related acquisition of bla ${ }_{\mathrm{NDM}}$-like genes in Acinetobacter baumannii," Antimicrobial Agents and Chemotherapy, vol. 56, no. 2, pp. 1087-1089, 2012.

[72] P. Nordmann, A. E. Boulanger, and L. Poirel, "NDM-4 metallo- $\beta$-lactamase with increased carbapenemase activity from Escherichia coli," Antimicrobial Agents and Chemotherapy, vol. 56, no. 4, pp. 2184-2186, 2012.

[73] M. Hornsey, L. Phee, and D. W. Wareham, "A novel variant, NDM- 5 , of the New Delhi metallo- $\beta$-lactamase in a multidrugresistant Escherichia coli ST648 isolate recovered from a patient in the United Kingdom," Antimicrobial Agents and Chemotherapy, vol. 55, no. 12, pp. 5952-5954, 2011.

[74] D. A. Williamson, H. E. Sidjabat, J. T. Freeman et al., "Identification and molecular characterisation of New Delhi metallo$\beta$-lactamase-1 (NDM-1)- and NDM-6-producing Enterobacteriaceae from New Zealand hospitals," International Journal of Antimicrobial Agents, vol. 39, no. 6, pp. 529-533, 2012.

[75] G. Cuzon, R. A. Bonnin, and P. Nordmann, "First identification of novel NDM carbapenemase, NDM-7, in Escherichia coli in France," PLoS ONE, vol. 8, no. 4, Article ID e61322, 2013.

[76] S. Gottig, A. G. Hamprecht, S. Christ, V. A. Kempf, and T. A. Wichelhaus, "Detection of NDM-7 in Germany, a new variant of the New Delhi metallo- $\beta$-lactamase with increased carbapenemase activity," Journal of Antimicrobial Chemotherapy, vol. 68, no. 8, pp. 1737-1740, 2013.

[77] T. Tada, T. Miyoshi-Akiyama, R. K. Dahal et al., "NDM-8 metallo- $\beta$-lactamase in a multidrug-resistant Escherichia coli strain isolated in Nepal," Antimicrobial Agents and Chemotherapy, vol. 57, no. 5, pp. 2394-2396, 2013.

[78] D. Girlich, L. Poirel, and P. Nordmann, "Value of the modified hodge test for detection of emerging carbapenemases in Enterobacteriaceae," Journal of Clinical Microbiology, vol. 50, no. 2, pp. 477-479, 2012.

[79] C. G. Carvalhaes, R. C. Picão, A. G. Nicoletti, D. E. Xavier, and A. C. Gales, "Cloverleaf test (modified Hodge test) for detecting carbapenemase production in Klebsiella pneumoniae: be aware of false positive results," The Journal of Antimicrobial Chemotherapy, vol. 65, no. 2, pp. 249-251, 2010.

[80] S. Bernabeu, L. Poirel, and P. Nordmann, "Spectrophotometrybased detection of carbapenemase producers among Enterobacteriaceae," Diagnostic Microbiology and Infectious Disease, vol. 74, no. 1, pp. 88-90, 2012.
[81] I. Burckhardt and S. Zimmermann, "Using matrix-assisted laser desorption ionization-time of flight mass spectrometry to detect carbapenem resistance within 1 to 2.5 hours," Journal of Clinical Microbiology, vol. 49, no. 9, pp. 3321-3324, 2011.

[82] J. Hrabak, E. Chudackova, and R. Walkova, "Matrix-assisted laser desorption ionization-time of flight (maldi-tof) mass spectrometry for detection of antibiotic resistance mechanisms: from research to routine diagnosis," Clinical Microbiology Reviews, vol. 26, no. 1, pp. 103-114, 2013.

[83] J. Hrabak, V. Studentova, R. Walkova et al., "Detection of NDM1, VIM-1, KPC, OXA-48, and OXA-162 carbapenemases by matrix-assisted laser desorption ionization-time of flight mass spectrometry," Journal of Clinical Microbiology, vol. 50, no. 7, pp. 2441-2443, 2012.

[84] J. Hrabák, R. Walková, V. Študentová, E. Chudáčková, and T. Bergerová, "Carbapenemase activity detection by matrixassisted laser desorption ionization-time of flight mass spectrometry," Journal of Clinical Microbiology, vol. 49, no. 9, pp. 3222-3227, 2011.

[85] M. Kempf, S. Bakour, C. Flaudrops et al., "Rapid detection of carbapenem resistance in Acinetobacter baumannii using matrix-assisted laser desorption ionization-time of flight mass spectrometry," PLoS ONE, vol. 7, no. 2, Article ID e31676, 2012.

[86] L. Dortet, L. Bréchard, L. Poirel, and P. Nordmann, "Comparison of diversegrowing media for further detection of carbapenemase production using the Carba NP test," Journal of Medical Microbiology, 2014. In press.

[87] P. Nordmann, L. Poirel, and L. Dortet, "Rapid detection of carbapenemase-producing Enterobacteriaceae," Emerging Infectious Diseases, vol. 18, no. 9, pp. 1503-1507, 2012.

[88] S. Vasoo, S. A. Cunningham, P. C. Kohner et al., "Comparison of a novel, rapid chromogenic biochemical assay, the Carba NP test with the modified Hodge test for detection of carbapenemase producing gram-negative bacilli," Journal of Clinical Microbiology, vol. 51, pp. 3097-3101, 2013.

[89] L. Dortet, L. Poirel, and P. Nordmann, "Rapid detection of carbapenemase-producing Pseudomonas spp," Journal of Clinical Microbiology, vol. 50, no. 11, pp. 3773-3776, 2012.

[90] L. Dortet, L. Poirel, and P. Nordmann, "Rapid identification of carbapenemase types in Enterobacteriaceae and Pseudomonas spp. by using a biochemical test," Antimicrobial Agents and Chemotherapy, vol. 56, no. 12, pp. 6437-6440, 2012.

[91] L. Dortet, L. Brechard, L. Poirel, and P. Nordmann, "Rapid detection of carbapenemase-producing Enterobacteriaceae from blood cultures," Clinical Microbiology and Infection, 2013.

[92] Y. Arakawa, N. Shibata, K. Shibayama et al., "Convenient test for screening metallo- $\beta$-lactamase-producing gram- negative bacteria by using thiol compounds," Journal of Clinical Microbiology, vol. 38, no. 1, pp. 40-43, 2000.

[93] P. Nordmann, L. Poirel, A. Carrër, M. A. Toleman, and T. R. Walsh, "How to detect NDM-1 producers," Journal of Clinical Microbiology, vol. 49, no. 2, pp. 718-721, 2011.

[94] T. R. Walsh, A. Bolmström, A. Qwärnström, and A. Gales, "Evaluation of a new Etest for detecting metallo- $\beta$-lactamases in routine clinical testing," Journal of Clinical Microbiology, vol. 40, no. 8, pp. 2755-2759, 2002.

[95] T. Naas, A. Ergani, A. Carredr, and P. Nordmann, "Real-time PCR for detection of NDM-1 carbapenemase genes from spiked stool samples," Antimicrobial Agents and Chemotherapy, vol. 55, no. 9, pp. 4038-4043, 2011.

[96] D. C. T. Ong, T.-H. Koh, N. Syahidah, P. Krishnan, and T. Y. Tan, "Rapid detection of the $b l a_{\mathrm{NDM}-1}$ gene by real-time PCR," 
Journal of Antimicrobial Chemotherapy, vol. 66, no. 7, pp. 16471649, 2011.

[97] G. Cuzon, T. Naas, P. Bogaerts, Y. Glupczynski, and P. Nordmann, "Evaluation of a DNA microarray for the rapid detection of extended-spectrum $\beta$-lactamases (TEM, SHV and CTX-M), plasmid-mediated cephalosporinases (CMY-2-like, DHA, FOX, ACC-1, ACT/MIR and CMY-1-like/MOX) and carbapenemases (KPC, OXA-48, VIM, IMP and NDM)," Journal of Antimicrobial Chemotherapy, vol. 67, no. 8, pp. 1865-1869, 2012.

[98] A. Adler, S. Navon-Venezia, J. Moran-Gilad, E. Marcos, D. Schwartz, and Y. Carmeli, "Laboratory and clinical evaluation of screening agar plates for detection of carbapenem-resistant Enterobacteriaceae from surveillance rectal swabs," Journal of Clinical Microbiology, vol. 49, no. 6, pp. 2239-2242, 2011.

[99] D. Landman, J. K. Salvani, S. Bratu, and J. Quale, "Evaluation of techniques for detection of carbapenem-resistant Klebsiella pneumoniae in stool surveillance cultures," Journal of Clinical Microbiology, vol. 43, no. 11, pp. 5639-5641, 2005.

[100] J. Moran Gilad, Y. Carmeli, D. Schwartz, and S. Navon-Venezia, "Laboratory evaluation of the CHROMagar KPC medium for identification of carbapenem-nonsusceptible Enterobacteriaceae," Diagnostic Microbiology and Infectious Disease, vol. 70, no. 4, pp. 565-567, 2011.

[101] D. Girlich, L. Poirel, and P. Nordmann, "Comparison of the SUPERCARBA, CHROMagar KPC, and Brilliance CRE screening media for detection of Enterobacteriaceae with reduced susceptibility to carbapenems," Diagnostic Microbiology and Infectious Disease, vol. 75, no. 2, pp. 214-217, 2013.

[102] K. M. Wilkinson, T. G. Winstanley, C. Lanyon, S. P. Cummings, M. W. Raza, and J. D. Perry, "Comparison of four chromogenic culture media for carbapenemase-producing Enterobacteriaceae," Journal of Clinical Microbiology, vol. 50, no. 9, pp. 31023104, 2012.

[103] K. M. Day, M. Salman, B. Kazi et al., "Prevalence of NDM1 carbapenemase in patients with diarrhoea in Pakistan and evaluation of two chromogenic culture media," Journal of Applied Microbiology, vol. 114, no. 6, pp. 1810-1816, 2013.

[104] P. Nordmann, D. Girlich, and L. Poirel, "Detection of carbapenemase producers in Enterobacteriaceae by use of a novel screening medium," Journal of Clinical Microbiology, vol. 50, no. 8, pp. 2761-2766, 2012.

[105] P. Nordmann and L. Poirel, "Strategies for identification of carbapenemase-producing Enterobacteriaceae," Journal of Antimicrobial Chemotherapy, vol. 68, no. 3, pp. 487-489, 2013. 

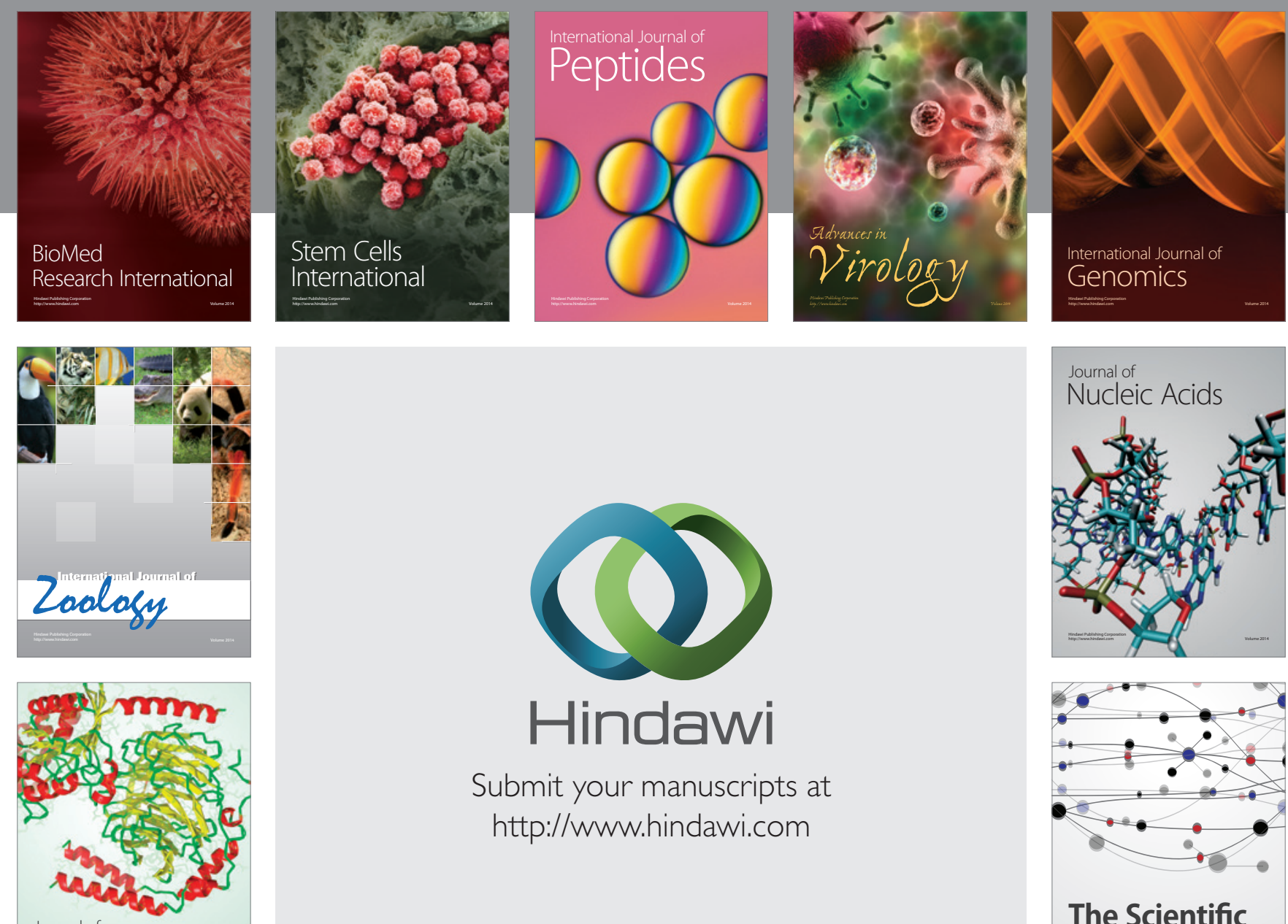

Submit your manuscripts at

http://www.hindawi.com

Journal of
Signal Transduction
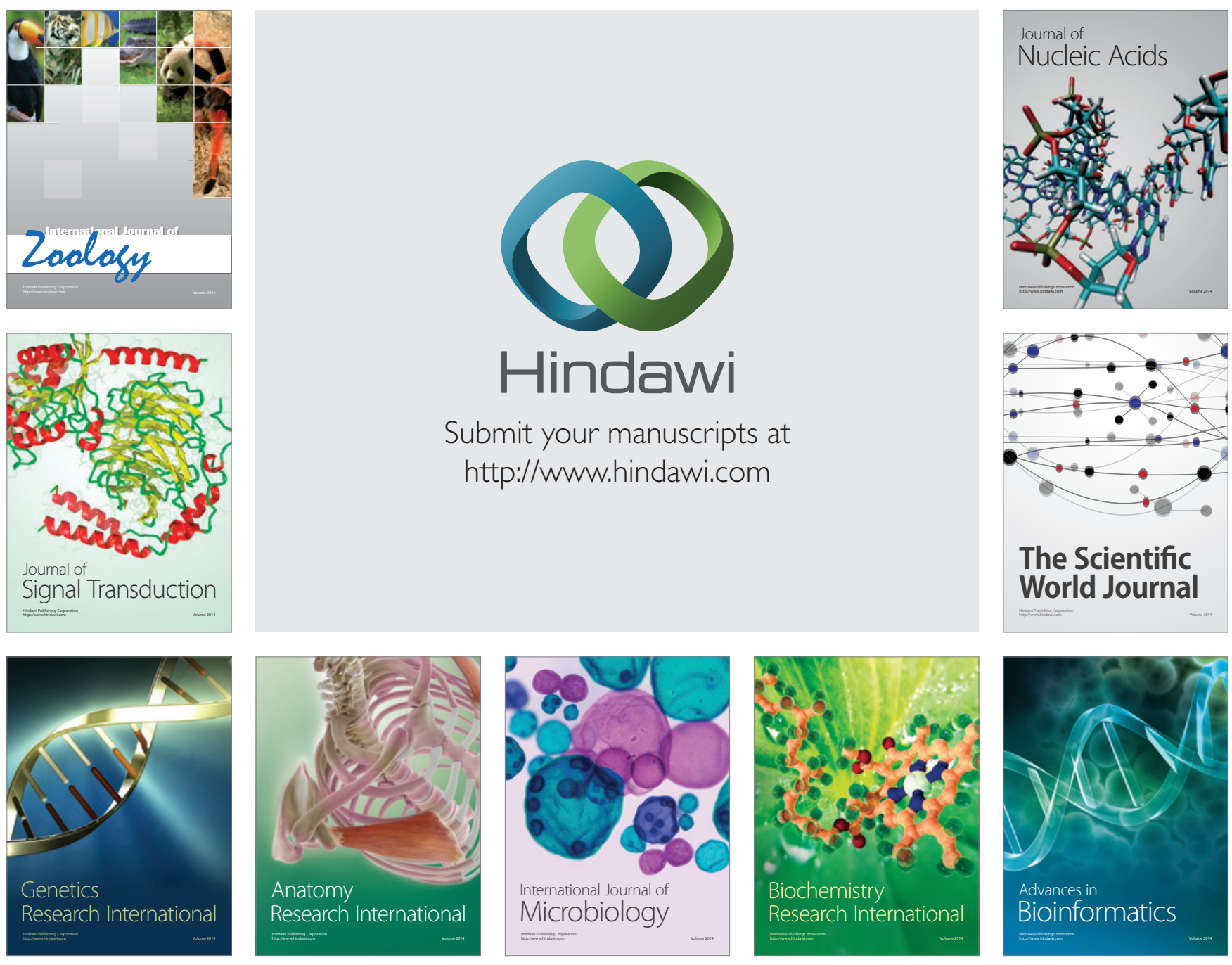

The Scientific World Journal
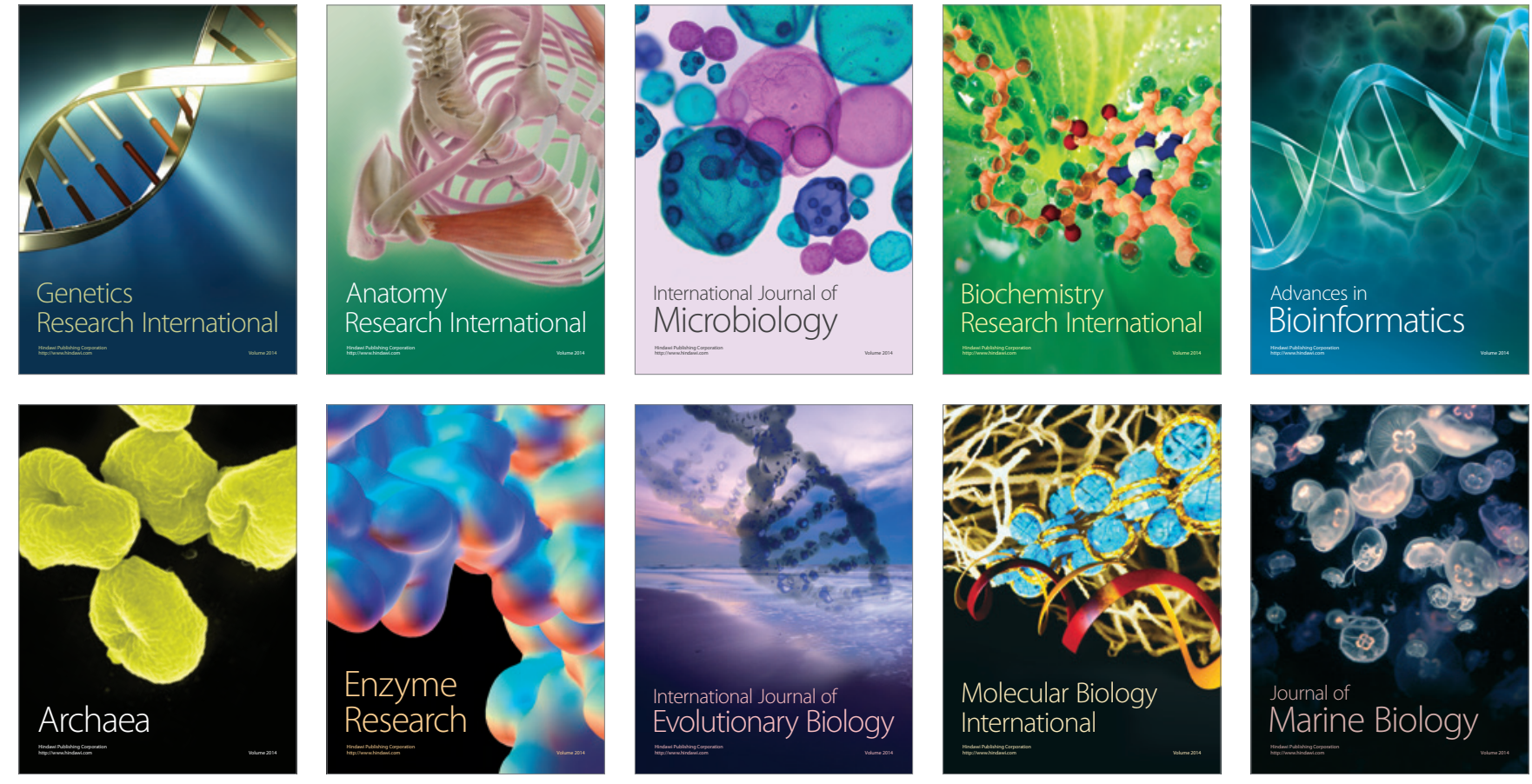